\title{
Unfallrisiko verschiedener Schichtsysteme - Simulation mit dem XIMES-Risikorechner
}

\author{
Anna ARLINGHAUS, Johannes GÄRTNER ${ }^{1}$ \\ XIMES GMbH, Wien \\ TU Wien
}

\begin{abstract}
Arbeitszeiten können das Fehler- und Unfallrisiko der Beschäftigten beeinflussen. Um die Risiken verschiedener Arbeitszeit- bzw. Schichtmodelle zu bewerten, wurden fünf häufig anzutreffende Schichtmodelle mit dem XIMES-Risikorechner analysiert und verglichen. Inkludiert wurden kurz vorwärts und lang rückwärts rotierte Schichtpläne, 12-Stunden-Modelle und lange Tagarbeit. Die Ergebnisse zeigen bei allen untersuchten Systemen erhöhte Risiken im Vergleich zu Tagarbeit mit 8-StundenSchichten, jedoch treten sowohl im Gesamtrisiko als auch in den Spitzen an einzelnen Arbeitstagen große Unterschiede auf. Das kurz vorwärts rotierte System schneidet dabei auf beiden Dimensionen am besten ab. Die Ergebnisse können für die Evaluierung und präventive Arbeitszeitgestaltung genutzt werden.
\end{abstract}

Keywords: Arbeitszeit, Schichtarbeit, Nachtarbeit, Unfallrisiko, Gefährdungsbeurteilung

\section{Einleitung}

Bei der arbeitswissenschaftlichen Bewertung von Arbeitstätigkeiten ist die Ausführbarkeit der Tätigkeit die Grundvoraussetzung, dass die Arbeit überhaupt ausgeübt werden kann, gefolgt von der Schädigungslosigkeit (Hacker/Richter 1984). Erstere und letztere sind nicht mehr gegeben, wenn z.B. das Unfallrisiko über eine Arbeitsschicht hinweg durch die Tätigkeit, die Umgebungsbedingungen oder die Organisation, z.B. die Arbeitszeitgestaltung signifikant erhöht sind. Gefährdungsanalysen sind in Deutschland gesetzlich vorgeschrieben. Sie können

\footnotetext{
${ }^{1}$ Dr. Anna Arlinghaus ist Arbeitspsychologin und arbeitet als Beraterin bei XIMES GmbH. Ehrenamtlich ist sie in den Vorständen der Working Time Society sowie der Arbeitszeitgesellschaft tätig: arlinghaus@ximes.com. Univ. Prof. Johannes Gärtner ist Gründer und Geschäftsführer der XIMES GmbH, unterrichtet an der Technischen Universität Wien und ist im Vorstand der Arbeitszeitgesellschaft tätig: gaertner@ximes.com.
}

DOI: http://dx.doi.org/10.18753/2297-8224-169 
Risiken sichtbar machen, jedoch führt nur etwa die Hälfte aller Betriebe in Deutschland eine Gefährdungsbeurteilung durch und nur ein Viertel berücksichtigt die Arbeitszeit dabei (BAuA 2016). Das deutet darauf hin, dass betrieblichen Praktiker*innen nicht immer klar ist, welche Risiken überhaupt bestehen können, wie man diese bewertet, oder dass nicht ausreichend geeignete Werkzeuge oder Ressourcen für eine solche Bewertung zur Verfügung stehen.

Zahlreiche empirische Studien zeigen, dass die Arbeitszeit einen Risikofaktor für Arbeitsunfälle und/oder Fehlhandlungen darstellen kann (z.B. Meta-Analysen von Fischer et al. 2017; Spencer, Robertson und Folkard 2006). In solchen Untersuchungen werden Arbeitszeiten in ihre Merkmale aufgeteilt und charakterisiert nach der Dauer, Lage, Verteilung und Dynamik der Arbeitszeit. Diese Merkmale werden wiederum auf Zusammenhänge mit arbeitsbedingten Unfällen geprüft, z.B. anhand von meldepflichtigen Unfällen, retrospektiven Befragungen von unfallbedingt ärztlich behandelten Personen oder querschnittlichen Fragebögen. Die Ergebnisse erlauben eine Abschätzung der Risiken, die sich aus Arbeitszeiten und konkreten Arbeitszeit- oder Schichtmodellen ergeben. Im Folgenden soll zunächst ein kurzer Überblick über die wichtigsten Risikofaktoren aus der Arbeitszeit gegeben werden, um darauf aufbauend den XIMES-Risikorechner und die mit diesem Tool bewerteten StandardSchichtpläne zu erläutern.

\section{Schichtarbeit mit Nachtarbeit}

Da der Mensch zirkadianen Rhythmen unterliegt, die auf Schlaf in der Nacht und Aktivität am Tag eingestellt sind, stört Arbeit zu biologisch ungünstigen Zeiten wie in der Nacht den Biorhythmus. Die Folgen sind erhöhte Müdigkeit und Erschöpfung während der Arbeit in der Nacht, kürzerer und schlechterer Schlaf während des Tages und ein langfristig erhöhtes Risiko für gesundheitliche Beeinträchtigungen (z.B. Moreno, Marqueze, Sargent, Wright, Ferguson und Tucker 2019). Die verringerte Leistungsfähigkeit in der Nacht führt zu einem höherem Fehler- und Unfallrisiko während der Arbeit, das sich empirisch in verschiedenen Studien nachweisen lässt (Fischer et al. 2017; Folkard, Lombardi und Spencer 2006; Spencer et al. 2006). Die Meta-Analyse von Fischer et al. (2017) ergibt zum Beispiel ein um 36\% erhöhtes Unfallrisiko für die Nachtschicht verglichen mit einer Frühschicht. Je mehr Schichten in Folge gearbeitet werden, umso größer wird das kumulative Schlafdefizit und damit auch das Unfallrisiko. Steigt es bei mehreren Frühschichten in Folge noch moderat um wenige Prozent an (4. Frühschicht in Folge: 9\% erhöhtes Risiko, nicht signifikant), lässt sich in der vierten Nachtschicht in Folge bereits ein um 36\% erhöhtes Risiko gegenüber der ersten Nachtschicht zeigen. Für Spätschichten sind die Zusammenhänge hingegen nicht eindeutig. Über die vierte Schicht in Folge hinaus liegen bislang für keine der Schichttypen ausreichend empirische Daten vor, es kann jedoch zumindest eine Fortsetzung des Trends über die ersten vier Schichten angenommen werden.

\section{Lange tägliche und wöchentliche Arbeitszeiten}

Tägliche Arbeitszeiten, die über 8-9 Stunden hinausgehen, sind mit einem erhöhten Unfallrisiko assoziiert. Ältere Studien wurden durch Fischer et al. (2017) bestätigt: Es zeigt sich ab der 9. Arbeitsstunde ein überproportional steigendes Risiko, das in der 12. Stunde bereits 
das Doppelte der 8. Stunde beträgt. Über 12 Stunden hinaus liegen nur unzureichend empirische Daten vor, jedoch kann angenommen werden, dass sich der exponentielle Trend fortsetzt (Spencer et al. 2006).

Die wöchentliche Arbeitszeit zeigt ebenfalls Zusammenhänge zum Unfallrisiko (Arlinghaus et al. 2012; Dembe et al. 2005; Folkard/Lombardi 2006), jedoch scheint hier eine Interaktion mit der Art und Länge der gearbeiteten Schichten vorzuliegen. Je länger die einzelnen Schichten sind, und je mehr die Arbeitszeitlage in biologisch ungünstige Zeiten fällt, desto höher das Risiko. Wie z.B. Folkard/Lombardi (2006) zeigten, entsteht so bei gleicher Wochenarbeitszeit von 48 Stunden ein deutlich niedrigeres Risiko, wenn sich diese Wochenarbeitszeit aus 6x8Stunden-Schichten am Tag zusammensetzt als beispielsweise bei $4 \times 12$-Stunden-Schichten in der Nacht.

\section{Kurze Ruhezeiten zwischen zwei Schichten}

Die Ruhezeit zwischen zwei Schichten muss ausreichen, um eine vollständige Erholung zu ermöglichen. Andernfalls wird die folgende Schicht mit bereits erhöhter Ermüdung und Erschöpfung begonnen, was wiederum zu einer erhöhten Beanspruchung durch die Arbeit führt, die dann eine noch längere Erholphase zur Rückführung auf den Ausgangswert benötigt (Effort-Recovery-Modell, vgl. Meijman/Mulder 1998). Gesetzlich ist für die meisten Beschäftigten eine minimale Ruhezeit von 11 Stunden festgeschrieben. In der Praxis kommen jedoch - neben Ausnahmeregelungen - auch kürzere Ruhezeiten vor, zum Beispiel durch kurz rückwärts rotierte Schichtsysteme, bei denen von der Nacht- zur Spät- und dann von der Spätzur Frühschicht gewechselt wird.

Die Frage, wie sich verkürzte Ruhezeiten auf das Unfallrisiko auswirken, ist noch nicht abschließend geklärt. Spencer et al. (2006) nehmen ein um 6\% erhöhtes Risiko für Ruhezeiten $<9$ Stunden an (das Risiko steigt mit jeder fehlenden Stunde Ruhezeit), jedoch basierte diese Schätzung damals auf eigenen Interpolationen. Neuere Ergebnisse finden sich im Review von Vedaa, Harris, Erevik, Waage, Bjorvatn, Sivertsen, Moen und Pallesen (2019) zu Schichtarbeit, Quick Returns und Unfällen bei Krankenpflegerinnen. Dort wird bereits bei $<11$ Stunden Ruhezeit ein erhöhtes Unfallrisiko berichtet. Beeinträchtigungen der Gesundheit und WorkLife-Balance bei Ruhezeiten von <11 Stunden werden auch von Backhaus et al. (2019) gezeigt. Die Schätzung von Spencer et al. (2006) scheint daher eher konservativ zu sein und berücksichtigt darüber hinaus, ebenso wie Backhaus, Brauner und Tisch (2019), noch nicht, welche Art von Schicht in welcher Länge zuvor gearbeitet wurde. Jedoch ist anzunehmen, dass der Erholungsbedarf nach belastenderen Arbeitszeiten größer ist als bei kurzen Einsätzen zu biologisch und sozial günstigen Zeiten. Blasche, Bauböck und Haluza (2017) zeigen zum Beispiel einen deutlich erhöhten Erholungsbedarf nach zwei 12-Stunden-Schichten: Erst nach drei Ruhetagen war die berichtete Ermüdung der Beschäftigten wieder auf dem Ausgangswert.

\section{Ausfall von Arbeitspausen}

Arbeitspausen sind notwendig, um bereits während der Arbeit die Beanspruchung durch Erholung auszugleichen, d.h. den Anstieg von Ermüdung und Erschöpfung im Laufe eines Arbeitstages zu kompensieren. Nicht nur für die Leistungsfähigkeit sind Pausen wertvoll; sie 
senken auch das Unfallrisiko, das sich aus mit der zunehmenden ununterbrochenen Arbeitszeit verminderter Leistungsfähigkeit oder Erschöpfung ergibt. Auch wenn die Studienlage zu Arbeitspausen und Unfallrisiken noch nicht sehr umfangreich ist (im Vergleich dazu gibt es bereits recht viele Studien zu den Auswirkungen von Pausen und Kurzpausen auf die Leistungsfähigkeit oder das Wohlbefinden, vgl. auch Wendsche/Lohmann-Haislah 2016), zeigt sich: Hinreichende Arbeitspausen können das vor der Pause bestehende Unfallrisiko um rund die Hälfte senken, wohingegen ein Ausfall von Pausen oder eine unzureichende Pausenanzahl bzw. -länge das gesamte Risiko erhöhen (Fischer et al. 2017; Spencer et al. 2006). Es scheint dabei einen Dosis-Wirkungs-Effekt zu geben, bei dem längere Pausen $>30$ Minuten das Risiko stärker senken als kürzere Pausen. Über eine Gesamtpausendauer von 60 Minuten hinaus lässt sich bisher jedoch keine weitere Reduktion des Risikos feststellen. Nach der Pause wirkt die Erholung nach und das Unfallrisiko steigt dann im Verlauf der nächsten Arbeitsstunden erst wieder an (Spencer et al. 2006).

\section{Bewertung von Unfallrisiken aus Arbeitszeitmodellen}

Die oben beschriebenen Erkenntnisse wurden von Spencer et al. (2006) in den Fatigue and Risk Index überführt, der mithilfe eines Excel-Tools die Unfallrisiken aus eingegebenen Arbeitszeiten berechenbar macht. Die zugrundeliegenden Algorithmen wurden von Fischer et al. (2017) aktualisiert, insbesondere im Bereich der Arbeitspausen und in neueren Studien zu den bisher verwendeten Risikofaktoren.

Um diese neueren Erkenntnisse für die Risikobewertung nutzbar zu machen und ein Tool auch für den deutschsprachigen Raum zur Verfügung zu stellen, wurde der XIMESRisikorechner entwickelt. Dieses webbasierte Software-Tool ermöglicht es, tatsächliche oder geplante Arbeitszeiten und Schichtmodelle hinsichtlich ihres Unfallrisikos zu bewerten. Es steht vonseiten der AUVA (Allgemeine Unfallversicherungsanstalt, Wien) für die nicht kommerzielle Nutzung für die Eingabe von maximal 100 Zeilen (Arbeitstagen) kostenlos zur Verfügung $^{2}$ und wird für Betriebe und die Beratung auch in einer kommerziellen Version angeboten. ${ }^{3}$ Eine ausführliche Beschreibung des Tools liegt von Gärtner et al. (2019) vor, daher wird im Folgenden nur ein verkürzter Überblick gegeben.

IST-Arbeitszeiten oder Dienstpläne können von Beschäftigten oder betrieblichen NutzerInnen in eine vorgegebene Erfassungsmaske mit Datum, Beginn und Ende jedes Arbeitstages sowie Pausen eingegeben oder hineinkopiert werden. Zusätzlich wird die tägliche Fahrtzeit zur Arbeit abgefragt. Der XIMES-Risikorechner schätzt dann auf Basis hinterlegter Rechenalgorithmen, um wie viel Prozent das Risiko des eingegebenen Arbeitszeitmodells höher oder niedriger liegt als das Risiko eines Referenz-Arbeitszeitmodells mit Tagarbeit (fünf Tage á 8 Stunden in Folge, inklusive 30 Minuten Pause und zwei freien Tagen im Anschluss) bei ansonsten gleichen Arbeitsbedingungen.

Die Berechnung des relativen Unfallrisikos beruht auf einer multiplikativen Kombination der folgenden, oben genauer beschriebenen Risikofaktoren:

\footnotetext{
${ }^{2}$ www.eval.at/evaluierung-arbeitszeit

${ }^{3}$ http://www.ximes.com/ximes-risikorechner
} 
- Schichtlänge: Das Unfallrisiko steigt nach der 8. Stunde überproportional an.

- Schichtart und Schichtfolge: Das Unfallrisiko steigt mit zunehmender Zahl aufeinanderfolgender Schichten. Nachtschichten erhöhen das Risiko mehr als Frühund Spätschichten.

- Pausen: Arbeitspausen reduzieren das Risiko, längere Pausen von mehr als 30 Minuten wirken dabei stärker risikosenkend als kürzere Pausen. Die Pausenwirkung schwächt sich in den Stunden nach der Pause wieder ab.

- Ruhezeit zwischen zwei Schichten: Liegen zwischen zwei aufeinanderfolgenden Schichten Ruhezeiten von weniger als 9 Stunden, erhöht sich das Risiko. Je kürzer die Ruhezeit, desto höher ist dabei das Risiko. Wurden zuvor Nachtschichten gearbeitet, wird mehr Ruhezeit benötigt, um das Risiko auf den Ausgangswert zu senken als bei Früh- oder Spätschichten.

- Wegezeiten: Werden mehr als 40 Minuten Fahrtzeit pro Weg angegeben, steigt das Risiko an. Je länger die Fahrtzeit, desto höher das Risiko (zusätzliche indirekte Wirkung: Fahrtzeiten werden nicht zur Arbeitszeit gezählt, reduzieren aber die Ruhezeit).

Diese Risikofaktoren und dazugehörige Gewichtungen wurden aus der Meta-Analyse von Fischer et al. (2017) und der vorherigen Version des Fatigue and Risk Index von Spencer et al. (2006) übernommen und - wenn keine meta-analytischen Schätzer vorlagen - durch eigene Interpolationen auf Basis arbeitswissenschaftlicher Theorien, ExpertInnenurteile und einzelner Studienergebnisse ergänzt.

Das Ergebnis ist ein Gesamtrisiko für das eingegebene Arbeitszeitmodell sowie eine Detailaufstellung der einzelnen Schichten und Stunden: Jeder eingegebene Arbeitstag erhält einen Risikowert, der aus den einzelnen Schätzern zu Fahrtzeit, Ruhezeit, Schichtfolge und Schichtlänge zusammensetzt ist.

\section{Fragestellung: Analyse verschiedener Standard-Schichtpläne}

Verschiedene Schichtmodelle können sich hinsichtlich aller oben genannter Arbeitszeitmerkmale unterscheiden und sollten dementsprechend auch unterschiedliche Risiken aufweisen. Um diese Hypothese zu prüfen, wurden verschiedene Schichtmodelle mit und ohne Nachtarbeit im XIMES-Risikorechner analysiert und ihre Risikobewertung für das gesamte Modell sowie für maximale Risiken einzelner Schichten verglichen. Das Ziel war, PraktikerInnen eine erste Orientierung zu geben, wie solche Standard-Schichtmodelle in der Risikobewertung abschneiden und welche Modelle aufgrund des Risikos bevorzugt verwendet werden sollten.

Folgende Schichtmodelle wurden inkludiert (eine ausführlichere Beschreibung unter Ergebnisse):

1. Ergonomisches System mit kurzer Vorwärtsrotation (2 Früh-, 2 Spät-, 2 Nachtschichten gefolgt von 3 freien Tagen)

2. Lang rückwärts rotiertes System mit 5 Nacht-, 5 Spät- und 5 Frühschichten mit 2 freien Tagen dazwischen 
3. 12-Stunden-Modell mit 1 Tag-, 1 Nachtschicht, gefolgt von 2 freien Tagen

4. 12-Stunden-Modell aus dem Offshore-Bereich mit 14 Tagen à 12 Stunden (7 Tag-, 7 Nachtschichten)

5. Lange Tagdienste mit 11-12 Stunden täglich, ohne Pausen

\section{Ergebnisse}

Im Folgenden werden die untersuchten Schichtsysteme genauer erläutert und die im Risikorechner ermittelten Schätzungen für das Unfallrisiko beschrieben. Es soll an dieser Stelle darauf hingewiesen werden, dass die Ergebnisse des Risikorechners auf einem Screeninglevel interpretiert werden sollten. Sie geben also einen Hinweis für das Unfallrisiko, das aufgrund des eingegebenen Schichtsystems im Vergleich zu Tagarbeit zu erwarten sein dürfte. Es handelt sich nicht um eine berufs- oder branchenspezifische Einschätzung und ebenfalls nicht um eine individuelle Risikoprognose für eine einzelne Person (da diese wiederum eine Vielzahl individueller Voraussetzungen mitbringt, die ihr eigenes Risiko a priori steigert oder senkt). Derartige Modelle sind aufgrund der bisher nicht hinreichend bekannten Wechselwirkungen zwischen Belastung- Beanspruchung und individuellen Merkmalen nicht realisierbar. Dies ist jedoch kein Nachteil, weil mit dem hier vorgestellten Rechner keine individuellen, belastungsbezogenen Prognosen erstellt werden sollen und für eine Gefährdungsbeurteilung auch nicht erforderlich sind.

\section{Kurz vorwärts rotiertes Schichtsystem mit 5 Gruppen}

Das Schichtmuster des kurz vorwärts rotierten Systems ist in Abbildung 1 dargestellt. Es handelt sich um ein System mit fünf Schichtgruppen, in denen alle Personen versetzt die Schichtfolge FFSSNN gefolgt von vier Tagen frei durchlaufen (F=Frühschicht von 6-14 Uhr, $\mathrm{S}=$ Spätschicht von 14-22 Uhr, N=Nachtschicht von 22-6 Uhr, jeweils inkl. 30 Minuten Pause). Die durchschnittliche Wochenarbeitszeit in diesem Modell beträgt 33:36 Std. inklusive Pausen.

\section{Abbildung 1: Kurz vorwärts rotiertes Schichtsystem mit 5 Gruppen}

\begin{tabular}{|c|c|c|c|c|c|c|c|c|c|c|c|c|c|c|}
\hline \multirow{2}{*}{ Gruppe } & \multicolumn{7}{|l|}{1} & \multicolumn{7}{|l|}{2} \\
\hline & Mo & $\mathrm{Di}$ & $\mathrm{Mi}$ & Do & $\mathrm{Fr}$ & Sa & So & Mo & $\mathrm{Di}$ & Mi & Do & $\mathrm{Fr}$ & Sa & So \\
\hline A & $\mathrm{F}$ & $F$ & $\mathrm{~S}$ & $S$ & N & N & & & & & $F$ & $\mathrm{~F}$ & $S$ & S \\
\hline B & $S$ & $S$ & N & N & & & & & $F$ & $F$ & $S$ & $S$ & N & $\mathbf{N}$ \\
\hline C & N & N & & & & & F & $F$ & S & S & N & N & & \\
\hline D & & & & & $\mathrm{F}$ & $\mathrm{F}$ & $S$ & $S$ & N & $\mathbf{N}$ & & & & \\
\hline $\mathbf{E}$ & & & $F$ & $F$ & S & S & $\mathrm{N}$ & $\mathrm{N}$ & & & & & $F$ & $F$ \\
\hline
\end{tabular}

Die Risikobewertung des kurz vorwärts rotierten Schichtsystems zeigt ein Durchschnittsrisiko von $8 \%$ gegenüber Tagarbeit (siehe Abbildung 2); das Schichtsystem ist also mit einem geringfügig erhöhten relativen Risiko verbunden. Die Spitze des Risikos wird in der 2. Nacht erreicht und beträgt ca. 44\%. Das Risiko ist damit zwar leicht erhöht aber insgesamt etwa vergleichbar mit dem von Tagarbeit mit 40 Wochenstunden bei sonst gleichen 
Arbeitsbedingungen. Werden nun zur Erhöhung der Wochenarbeitszeit Einbring- oder Zusatzschichten hinzugefügt, wird das Risiko - je nach Schichtart und -abfolge - etwas steigen.

Abbildung 2: Kurz vorwärts rotiertes Schichtsystem mit 5 Gruppen - Unfallrisikoänderung

\section{Das relative Unfallrisiko ändert sich um:}

Das Risiko wird im Verhältnis zum durchschnittlichen Risiko eines Referenz-Arbeitszeitmodells mit 5

Tagen Frühschicht mit je 8 Stunden Arbeit und 30 Minuten Pause gefolgt von zwei Tagen frei berechnet = REFERENZRISIKO.

Die Veränderung des relativen Unfallrisikos pro Arbeitstag im Zeitverlauf:

In der Grafik werden Werte einer Arbeitszeit dem Tag zugeordnet, an dem die Arbeitszeit beginnt. Freie Tage werden nicht angezeigt.

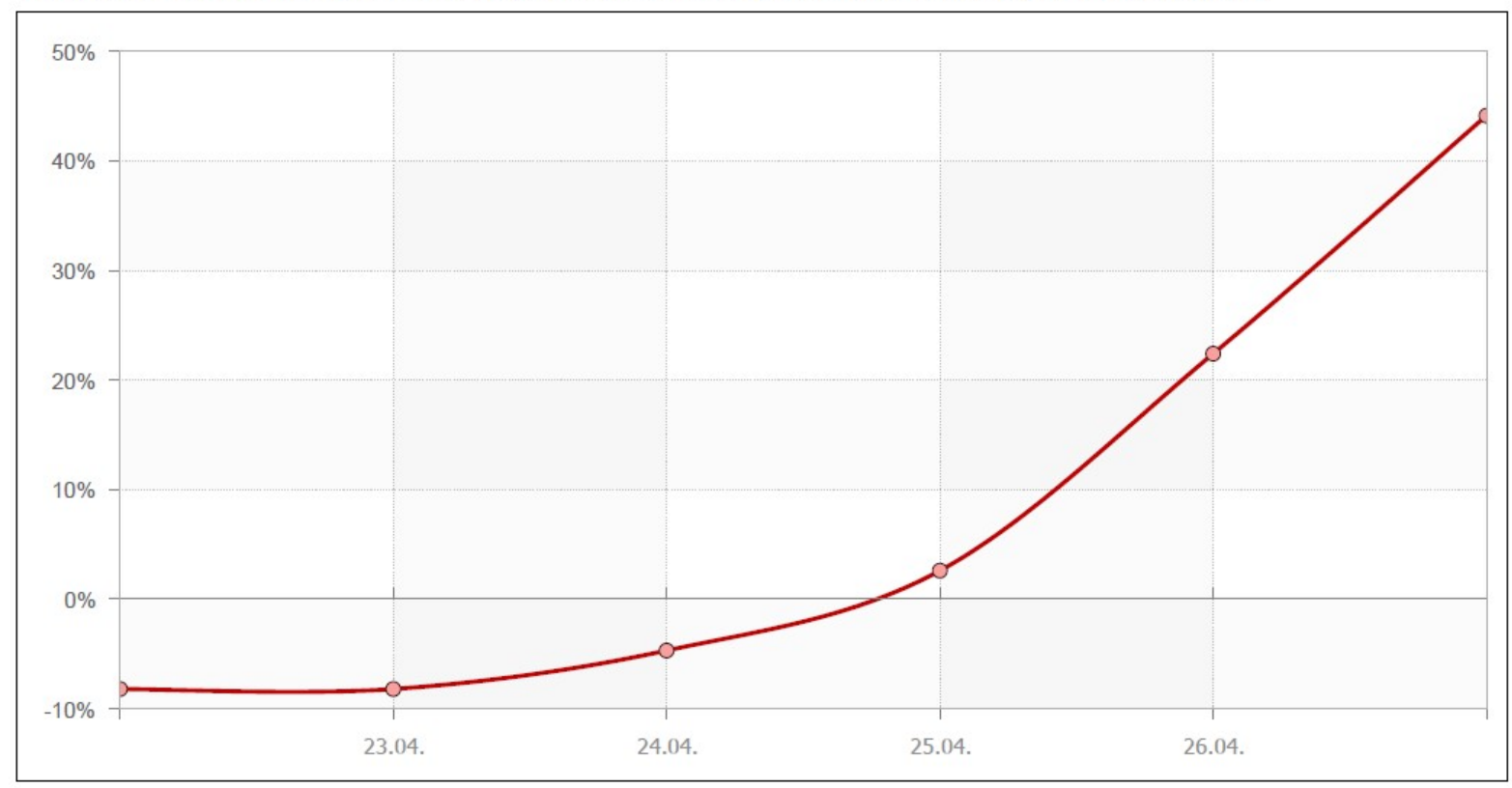

Lang rückwärts rotierendes teilkontinuierliches 3-Gruppen Schichtsystem

Das Schichtmuster des lang rückwärts rotierten Systems ist in Abbildung 3 dargestellt. Es handelt sich um ein System mit drei Schichtgruppen, in denen alle Personen versetzt fünf Tage Arbeit gefolgt von zwei Tagen frei im Rhythmus Nacht - Spät - Früh durchlaufen (F=Frühschicht von 6-14 Uhr, S=Spätschicht von 14-22 Uhr, N=Nachtschicht von 22-6 Uhr, jeweils inkl. 30 Minuten Pause). Die durchschnittliche Wochenarbeitszeit in diesem Modell beträgt $40 \mathrm{Std}$. inklusive Pausen.

Abbildung 3: Lang rückwärts rotierendes teilkontinuierliches 3-Gruppen Schichtsystem

\begin{tabular}{|c|c|c|c|c|c|c|c|}
\hline \multirow{2}{*}{ Gruppe } & \multicolumn{7}{|l|}{1} \\
\hline & Mo & Di & Mi & Do & $\mathrm{Fr}$ & Sa & So \\
\hline A & N & N & N & N & N & & \\
\hline B & S & S & S & S & $S$ & & \\
\hline C & $\mathrm{F}$ & $\mathrm{F}$ & $\mathrm{F}$ & $\mathrm{F}$ & $\mathrm{F}$ & & \\
\hline
\end{tabular}


Die Risikobewertung dieses Systems (Abbildung 4) ergibt ein Durchschnittsrisiko von 19\% über den gesamten Schichtzyklus hinweg. Die Risikospitze tritt in der fünften Nacht auf und beträgt $88 \%$ gegenüber einer Tagschicht. Insbesondere der lange Nachtblock am Anfang trägt zu dem insgesamt erhöhten Risiko bei.

Abbildung 4: Lang rückwärts rotierendes teilkontinuierliches 3-Gruppen Schichtsystem - Unfallrisikoänderung

Das relative Unfallrisiko ändert sich um:

Das Risiko wird im Verhältnis zum durchschnittlichen Risiko eines Referenz-Arbeitszeitmodells mit 5

Tagen Frühschicht mit je 8 Stunden Arbeit und 30 Minuten Pause gefolgt von zwei Tagen frei berechnet = REFERENZRISIKO.

Die Veränderung des relativen Unfallrisikos pro Arbeitstag im Zeitverlauf: In der Grafik werden Werte einer Arbeitszeit dem Tag zugeordnet, an dem die Arbeitszeit beginnt. Freie Tage werden nicht angezeigt.

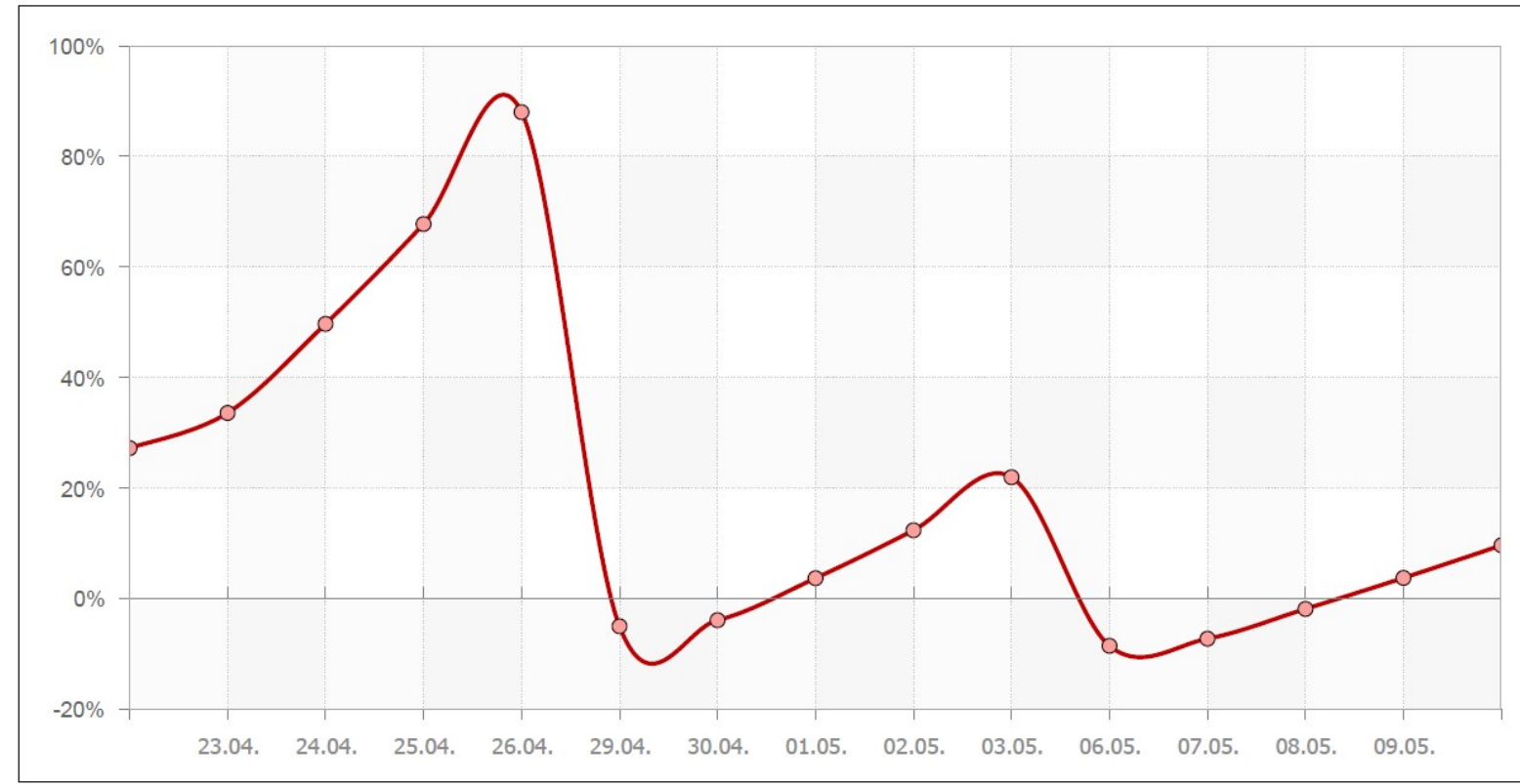

\section{2-Stunden-Modell mit zwei Schichten in Folge}

Das Schichtmuster des 12-Stunden-Modells ist in Abbildung 5 dargestellt. Es handelt sich um ein System mit vier Schichtgruppen, in denen alle Personen versetzt eine zwölfstündige Tagschicht (T, von 6-18 Uhr) gefolgt von einer zwölfstündigen Nachtschicht am folgenden Tag (N, von 18-6 Uhr) und im Anschluss zwei freien Tagen (48 Stunden) arbeiten. Die Schichten beinhalten jeweils 45 Minuten Pause. Die durchschnittliche Wochenarbeitszeit in diesem Modell beträgt 42 Std. inklusive Pausen. 
Abbildung 5: 12-Stunden-Modell mit zwei Schichten in Folge

\begin{tabular}{|c|c|c|c|c|c|c|c|}
\hline \multirow{2}{*}{ Gruppe } & \multicolumn{7}{|l|}{1} \\
\hline & Mo & Di & Mi & Do & $\mathrm{Fr}$ & $\mathrm{Sa}$ & So \\
\hline A & $\mathrm{T}$ & N & & & $T$ & N & \\
\hline B & & $T$ & N & & & $T$ & N \\
\hline C & & & $T$ & N & & & $T$ \\
\hline D & N & & & $\mathrm{T}$ & N & & \\
\hline
\end{tabular}

Die Risikobewertung (Abbildung 6) ergibt ein Durchschnittsrisiko von 39\% mit einem maximalen Risiko von 54\% in den Nachtschichten. Da dieses Risiko offenbar in der folgenden Ruhezeit abgebaut werden kann, findet keine Kumulation des Risikos statt. Durch gegebenenfalls notwendige Freischichten zur Erzielung einer niedrigeren Wochenarbeitszeit kann sich das Risiko punktuell verringern.

Abbildung 6: 12-Stunden-Modell mit zwei Schichten in Folge - Unfallrisikoänderung

Das relative Unfallrisiko ändert sich um:

Das Risiko wird im Verhältnis zum durchschnittlichen Risiko eines Referenz-Arbeitszeitmodells mit 5

Tagen Frühschicht mit je 8 Stunden Arbeit und 30 Minuten Pause gefolgt von zwei Tagen frei berechnet $=$

REFERENZRISIKO.

Die Veränderung des relativen Unfallrisikos pro Arbeitstag im Zeitverlauf:

In der Grafik werden Werte einer Arbeitszeit dem Tag zugeordnet, an dem die Arbeitszeit beginnt. Freie Tage werden nicht angezeigt.

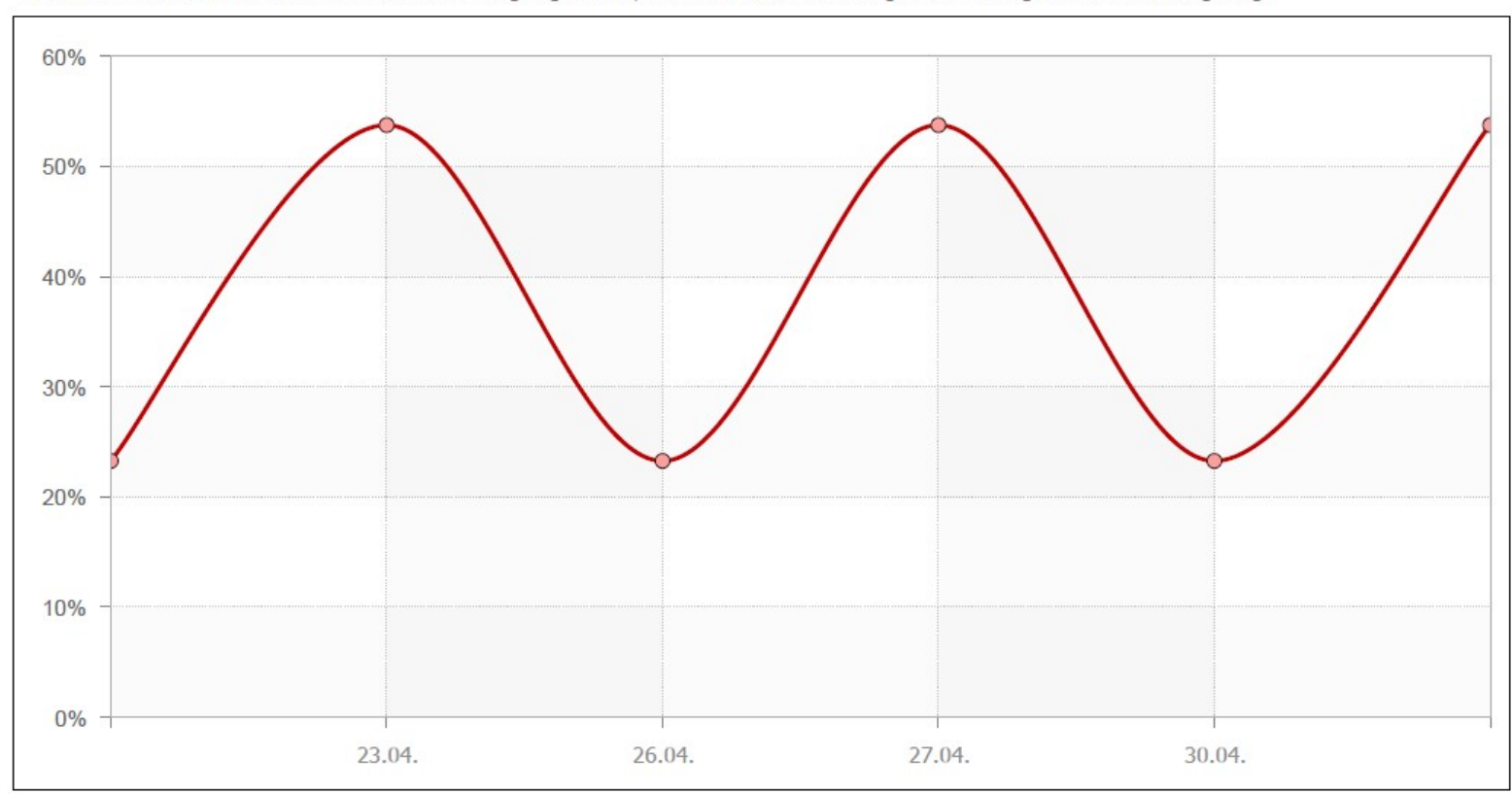

Offshore-Modell mit 14 Schichten in Folge

Bei diesem Schichtsystem handelt es sich um ein häufig in der Offshore-Industrie gefahrenes Modell, bei dem die Beschäftigten aufgrund der meist langen An- und Abfahrt zum Arbeitsplatz und der dortigen Unterbringung sieben 12-stündige Tagschichten gefolgt von sieben 12stündigen Nachtschichten arbeiten und danach 14 Tage frei haben (Abbildung 7). Die Schichten beinhalten jeweils 45 Minuten Pause. Die durchschnittliche Wochenarbeitszeit in diesem Modell beträgt 42 Std. inklusive Pausen. 
Abbildung 7: Offshore-Modell mit 14 Schichten in Folge

\begin{tabular}{|c|l|l|l|l|l|l|l}
\multirow{2}{*}{ Gruppe } & 1 & \multicolumn{1}{|l}{} \\
\cline { 2 - 8 } & Mo & Di & Mi & Do & Fr & Sa & So \\
\hline A & T & T & T & T & T & T & T \\
\hline B & N & N & N & N & N & N & N \\
\hline C & & & & & & & \\
\hline D & & & & & & & \\
\hline
\end{tabular}

Die Risikobewertung für dieses System ergibt ein Durchschnittsrisiko von 124\% (Abbildung 8). Das Risiko steigt kontinuierlich mit jeder zusätzlichen Schicht an, wobei der Anstieg während des Nachtschichtblocks steiler ausfällt als während des Tagschichtblocks. Die Risikospitze in der letzten Nacht liegt bei 342\%. Es ist daher zu erwarten, dass eine sehr lange Erholphase notwendig ist um die Belastung vollständig abzubauen, was den Wert des langen Freizeitblocks deutlich verringern dürfte.

Abbildung 8: Offshore-Modell mit 14 Schichten in Folge - Unfallrisikoänderung

Das relative Unfallrisiko ändert sich um:

Das Risiko wird im Verhältnis zum durchschnittlichen Risiko eines Referenz-Arbeitszeitmodells mit 5

Tagen Frühschicht mit je 8 Stunden Arbeit und 30 Minuten Pause gefolgt von zwei Tagen frei berechnet REFERENZRISIKO.

Die Veränderung des relativen Unfallrisikos pro Arbeitstag im Zeitverlauf:

In der Grafik werden Werte einer Arbeitszeit dem Tag zugeordnet, an dem die Arbeitszeit beginnt. Freie Tage werden nicht angezeigt.

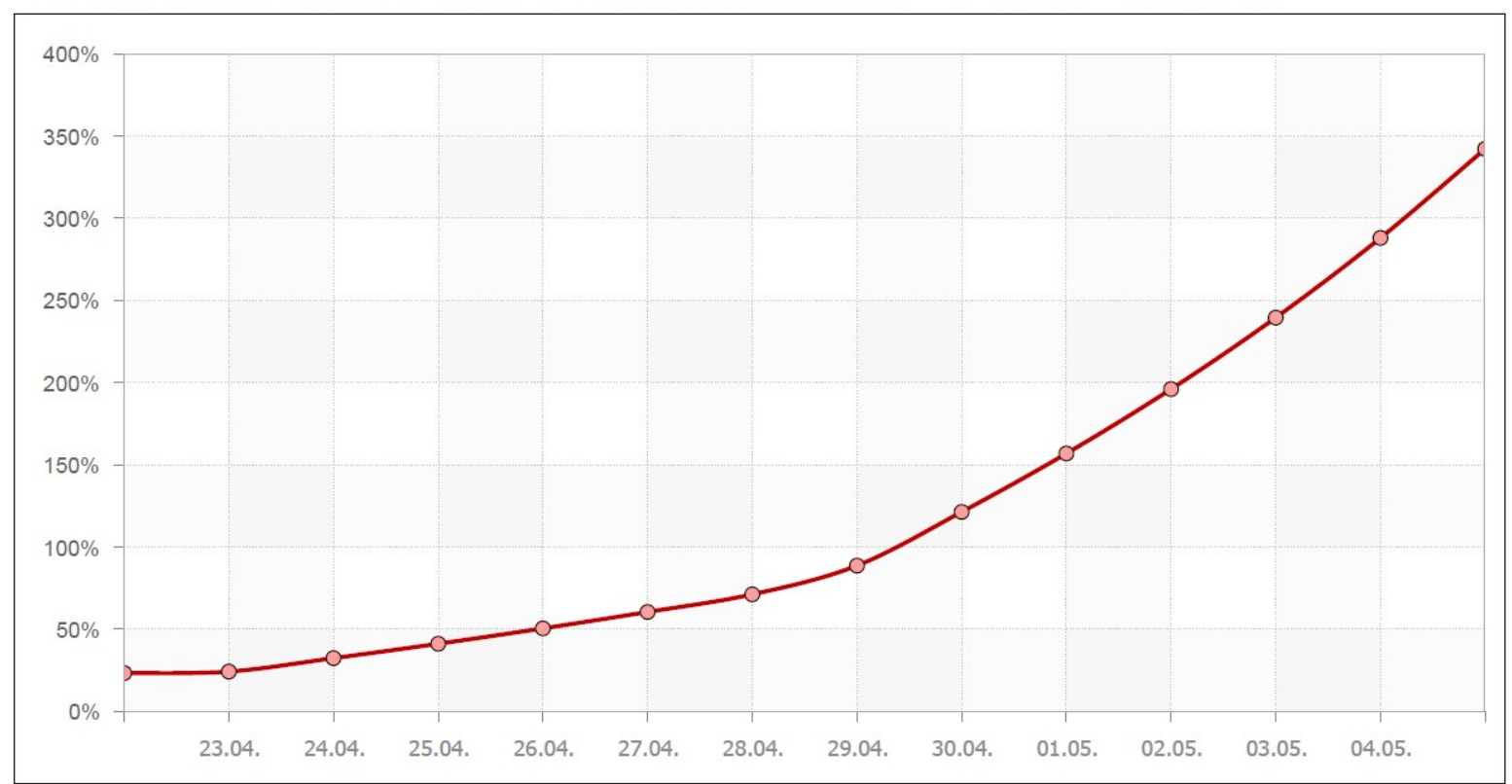

\section{Beispiel für Lange Tagdienste}

Als Ergänzung zu den eher klassischen Schichtsystemen oben wurde zusätzlich ein Modell mit fünf langen Arbeitstagen ohne Pause sowie einem kürzeren sechsten Arbeitseinsatz simuliert (siehe Abbildung 9). Die wöchentliche Arbeitszeit beträgt hier 56:30 Stunden. 
Abbildung 9: Beispiel für Lange Tagdienste
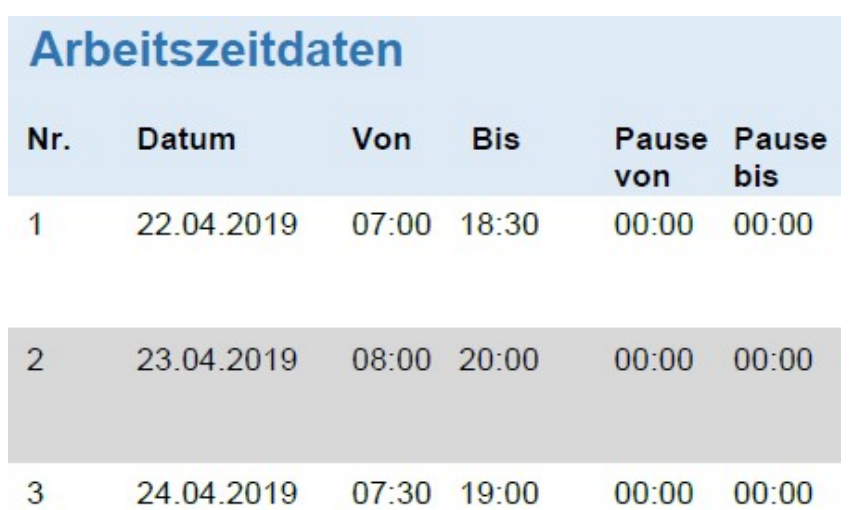

\begin{tabular}{|llllll|}
\hline 4 & 25.04 .2019 & $08: 00$ & $18: 30$ & $00: 00$ & $00: 00$ \\
\hline 5 & 26.04 .2019 & $07: 00$ & $18: 00$ & $00: 00$ & $00: 00$ \\
\hline 6 & 27.04 .2019 & $07: 00$ & $12: 00$ & $00: 00$ & $00: 00$ \\
\hline
\end{tabular}

Die Risikobewertung ergibt ein Durchschnittsrisiko von 43\%, wobei die täglichen Risikoschätzer je nach Länge des Arbeitstages zwischen 33\% und 60\% schwanken (Abbildung 10). Aufgrund der langen Einsätze ist das Risiko gegenüber 8-Stunden-Schichten deutlich erhöht und das durchschnittliche Risiko ist etwa vergleichbar mit dem obigen 12-StundenSchichtmodell, in dem immer zwei Tage in Folge gearbeitet werden. 
Abbildung 10: Beispiel für Lange Tagdienste - Unfallrisikoänderung

Das relative Unfallrisiko ändert sich um:

Das Risiko wird im Verhältnis zum durchschnittlichen Risiko eines Referenz-Arbeitszeitmodells mit 5 Tagen

Frühschicht mit je 8 Stunden Arbeit und 30 Minuten Pause gefolgt von zwei Tagen frei berechnet =

REFERENZRISIKO.

Das relative Unfallrisiko pro Arbeitstag im Zeitverlauf:

In der Grafik werden Werte einer Arbeitszeit dem Tag zugeordnet, an dem die Arbeitszeit beginnt. Freie Tage werden nicht angezeigt.

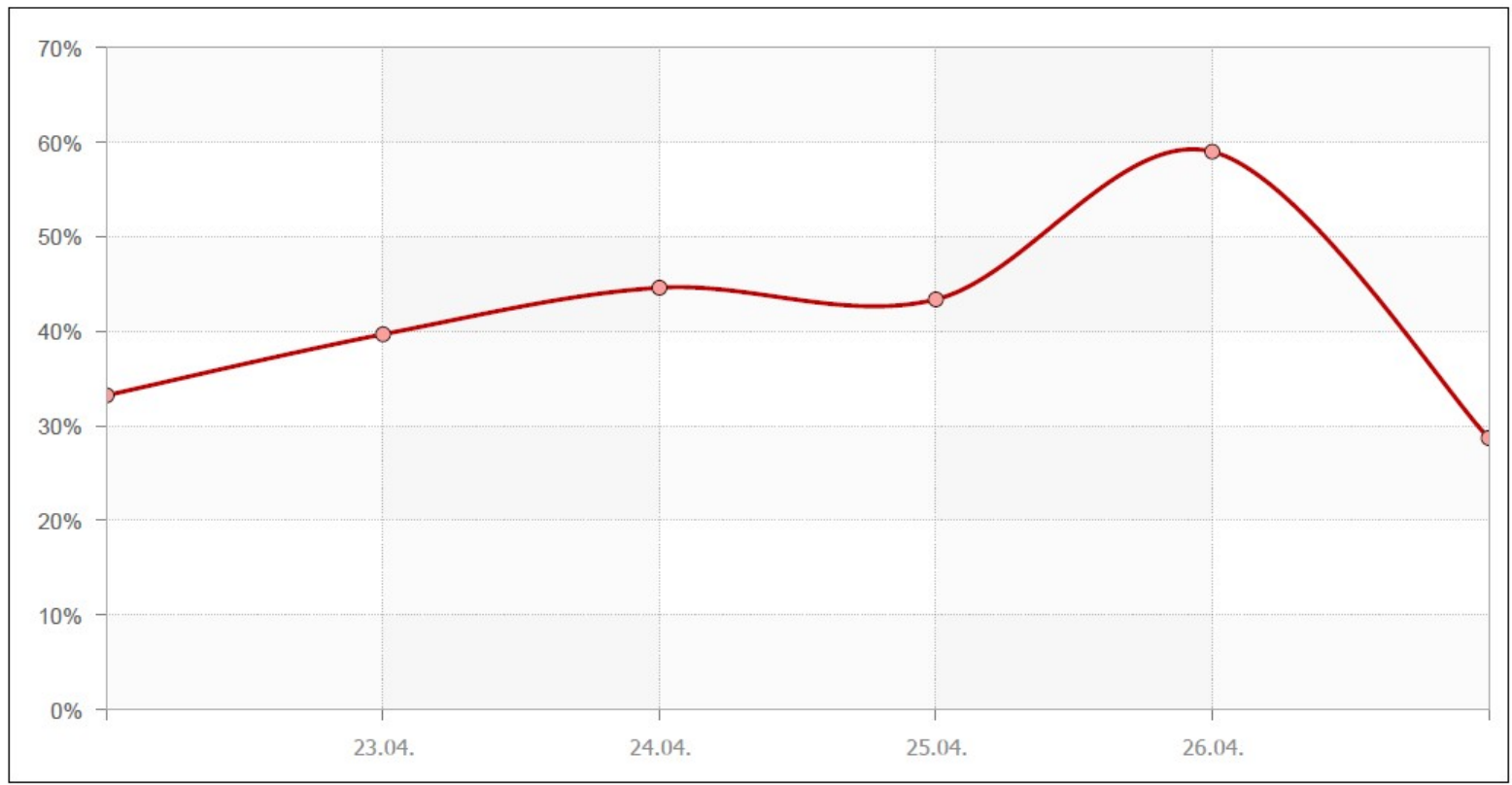

\section{Diskussion}

Die Ergebnisse der Simulationsrechnungen zeigen bei allen untersuchten Systemen höhere Risiken im Vergleich zu normaler Tagarbeit (5 Tage à 8 Stunden), jedoch treten abhängig vom Schichtsystem große Unterschiede auf: Das kurz vorwärts rotierte Schichtsystem, welches dem aktuellen Stand der arbeitswissenschaftlichen Erkenntnisse entspricht, schneidet mit einem durchschnittlich um 8\% erhöhtem Risiko am günstigsten ab. Einerseits wirkt die kurze Wochenarbeitszeit günstig, vor allem wird aber ein Risikoanstieg vermieden durch die kurze Rotation mit maximal zwei Nachtschichten in Folge mit ausreichender anschließender Ruhezeit. Das lang rückwärts rotierte System führt hingegen zu einem um 19\% und die 12Stunden-Modelle zu einem um 39\% (Tag- und Nachtschichten) bzw. 43\% (viele lange Tagschichten in Folge) erhöhten Risiko. Das Offshore-Modell weist das höchste Risiko mit einer durchschnittlichen Erhöhung um 124\% auf.

Auch die maximalen Risiken für einzelne Arbeitstage zeigen, dass das ergonomische System mit einer Spitze von ca. 45\% für die letzte Nachtschicht am günstigsten abschneidet. Einzelne 12-stündige Nachtschichten erreichen 54\%, die fünfte Nacht in Folge im lang rückwärts rotierten System 88\% und die 14. Schicht (7. Nacht) in Folge im Offshore-Modell bereits 342\% Risikoerhöhung. 
Offensichtlich gibt es also deutliche Unterschiede zwischen den verschiedenen Systemen sowohl in der Gesamt-Risikobilanz als auch in den maximalen Risiken an einzelnen Tagen. Die arbeitswissenschaftlichen Empfehlungen (z.B. Rothe, Beermann und Wöhrmann 2017), möglichst wenige Nachtschichten in Folge zu arbeiten, die Dauer der täglichen und wöchentlichen Arbeitszeit zu begrenzen und adäquate Erholungspausen sowie Ruhezeiten zwischen den Schichten zu gewähren, spiegeln sich in diesem Risikomodell wider, das auf Basis empirischer Daten zu Arbeitszeiten und Unfällen erstellt wurde. Die zugrundeliegenden Schätzer sind anhand vieler empirischer Studien und Meta-Analysen abgesichert und liefern den theoretischen Annahmen entsprechende Ergebnisse. Auch wenn die Risikobewertungen hier auf einem Screeninglevel erfolgen, lässt sich dennoch eine gute Übersicht über verschiedene Modelle generieren, die bei der Bewertung, Auswahl und Optimierung von Arbeitszeitsystemen unterstützen kann.

Noch nicht berücksichtigt werden Faktoren wie die Arbeitsbelastung, die über eine oder mehrere Schichten hinweg mit der Arbeitszeit interagieren, z.B. indem eine körperlich und/oder mental hoch belastende Tätigkeit über 12 Stunden hinweg einen überproportional stärkeren Risikoanstieg verursacht als eine weniger belastende Tätigkeit. Ebenso liegen noch keine stabilen Algorithmen für Bereitschaftsdienste, geteilte Dienste oder Ruhezeiten zwischen 9 und 11 Stunden vor (da die Studie von Vedaa et al., 2019 erst nach Entwicklung des Risikorechners publiziert wurde, konnten die Ergebnisse noch nicht in die aktuelle Version integriert werden). Auch zur Dynamik, also der Abfolge von Arbeits- und Ruhezeiten, konnten keine empirischen Daten einfließen.

Individuelle Faktoren wie das Schlafverhalten, Geschlecht oder Alter werden ebenfalls nicht inkludiert, da für diese Subgruppen nur unzureichende empirische Daten vorliegen und von einer individuellen Risikoprognose aufgrund der großen Streubreiten bei solchen Vorhersagen Abstand genommen wurde (vgl. Gärtner et al. 2019) und diese für eine generalisierte Gefährdungsanalyse nicht erforderlich ist.

\section{Fazit}

Der XIMES-Risikorechner ermöglicht eine rasche Bewertung von durchschnittlichen und maximalen Risiken verschiedener Schichtmodelle. Die Ergebnisse können für die Evaluierung oder Gefährdungsbeurteilung von Arbeitszeiten oder für die präventive Arbeitszeitgestaltung genutzt werden.

Offene Forschungsfragen wie z.B zu den Auswirkungen von Rufbereitschaften, geteilten Diensten oder der Integration von Belastungsaspekten durch die Tätigkeit sind derzeit noch nicht implementiert. Der open-source Ansatz für die Wissenschaft ermöglicht jedoch Ergänzungen und Modifikationen wenn neue empirische Erkenntnisse vorliegen.

\section{Danksagung}

Die AutorInnen bedanken sich bei den Redaktoren des Journals sozialpolitik.ch für die wertvollen Hinweise und Kommentare. 


\section{Deklaration von Interessenkonflikten}

Die AutorInnen deklarieren keine Interessenkonflikte in Bezug auf Forschung, AutorInnenschaft und/oder Publikation des Artikels.

\section{Finanzierung}

Die AutorInnen haben keine finanzielle Unterstützung für die Forschung, die AutorInnenschaft und die Publikation dieses Artikels erhalten

\section{Bibliographie}

Arlinghaus, Anna, David A. Lombardi, Joanna L. Willetts, Simon Folkard und David C. Christiani (2012). A structural equation modeling approach to fatigue-related risk factors for occupational injury. American Journal of Epidemiology 176(7), 597-607.

Backhaus, Nils, Corinna Brauner und Anita Tisch (2019). Auswirkungen verkürzter Ruhezeiten auf Gesundheit und Work-Life-Balance bei Vollzeitbeschäftigten: Ergebnisse der BAuA-Arbeitszeitbefragung 2017. Zeitschrift für Arbeitswissenschaft 73(4), 394-417.

BAuA (2016). Arbeitswelt im Wandel. Zahlen - Daten - Fakten. Dortmund: Bundesanstalt für Arbeitsschutz und Arbeitsmedizin. Zugriff am 22.11.2019 auf https://www.baua.de/DE/Angebote/Publikationen/Praxis/A95.pdf? blob=publicationFile\&v=2

Blasche, Gerhard, Verena-Maria Bauböck und Daniela Haluza (2017). Work-related self-assessed fatigue and recovery among nurses. International Archives of Occupational and Environmental Health 90(2), 197-205.

Dembe, Allard, J. Bianca Erickson, Rachel G. Delbos, Steven M. Banks (2005). The impact of overtime and long work hours on occupational injuries and illnesses: new evidence from the United States. Occupational and Environmental Medicine 62(9), 588-597.

Fischer, Dorothee, David A. Lombardi, Simon Folkard, Joanna Willetts und David C. Christiani (2017). Updating the "Risk Index": A systematic review and meta-analysis of occupational injuries and work schedule characteristics. Chronobiology International 34(10), 1423-1438.

Folkard, Simon, David A. Lombardi und Mick B. Spencer (2006). Estimating the circadian rhythm in the risk of occupational injuries and accidents. Chronobiology International 23(6), 1181-1192.

Folkard, Simon und David A. Lombardi (2006). Modeling the Impact of the Components of Long Work Hours on Injuries and “Accidents". American Journal of Industrial Medicine 49(11), 953-963.

Gärtner, Johannes, Anna Arlinghaus, Magdalena Widl und Werner Schafhauser (2019). Ein neues Tool zur Bewertung von Arbeitszeiten. Sichere Arbeit 4/2019, 11-15. Zugriff am 20.11 .2019 auf http://www.sicherearbeit.at/cms/X04/X04 0.a/1342614881024/home/ein-neues-tool-zur-bewertung-von-arbeitszeiten

Hacker, Winfried und Peter Richter (1984). Psychologische Bewertung von Arbeitsgestaltungsmaßnahmen - Ziele und Bewertungsmaßstäbe. Berlin: VEB Deutscher Verlag der Wissenschaften. 
Meijman, Theo F. und Gijsbertus Mulder (1998). Psychological aspects of workload. In: Drenth, Pieter J.D., Henk Thierry und Charles de Wolff (Hg.). Handbook of work and organizational psychology: Vol. 2. Work psychology. Hove, UK: Psychology Press, 5-33.

Moreno, Claudia R.C., Elaine C. Marqueze, Charli Sargent, Kenneth P. Wright Jr, Sally A. Ferguson, Philip Tucker (2019). Working Time Society consensus statements: Evidence-based effects of shift work on physical and mental health. Industrial Health 57(2), 139-157.

Rothe, Isabell, Beate Beermann und Anne Marit Wöhrmann (2017). Arbeitswissenschaftliche Erkenntnisse zu Arbeitszeit und Gesundheit. In: Schröder, Lothar und Hans-Jürgen Urban (Hg.). Gute Arbeit. Streit um Zeit - Arbeitszeit und Gesundheit. Frankfurt am Main: Bund-Verlag, S. 123-135.

Spencer, Mick, Karen Robertson und Simon Folkard (2006). The development of a fatigue/risk index for shiftworkers. Health and Safety Executive Research Report 446. Zugriff am 20.11.2019 auf http://www.hse.gov.uk/research/rrhtm/rr446.htm.

Vedaa, Øystein, Anette Harris, Eilin K. Erevik, Siri Waage, Bjørn Bjorvatn, Børge Sivertsen, Bente E. Moen und Ståle Pallesen (2019). Short rest between shifts (quick returns) and night work is associated with work-related accidents. International Archives of Occupational and Environmental Health 92(6), 829-835.

Wendsche, Johannes und Andrea Lohmann-Haislah (2016). Psychische Gesundheit in der Arbeitswelt: Pausen. Dortmund: Bundesanstalt für Arbeitsschutz und Arbeitsmedizin. 